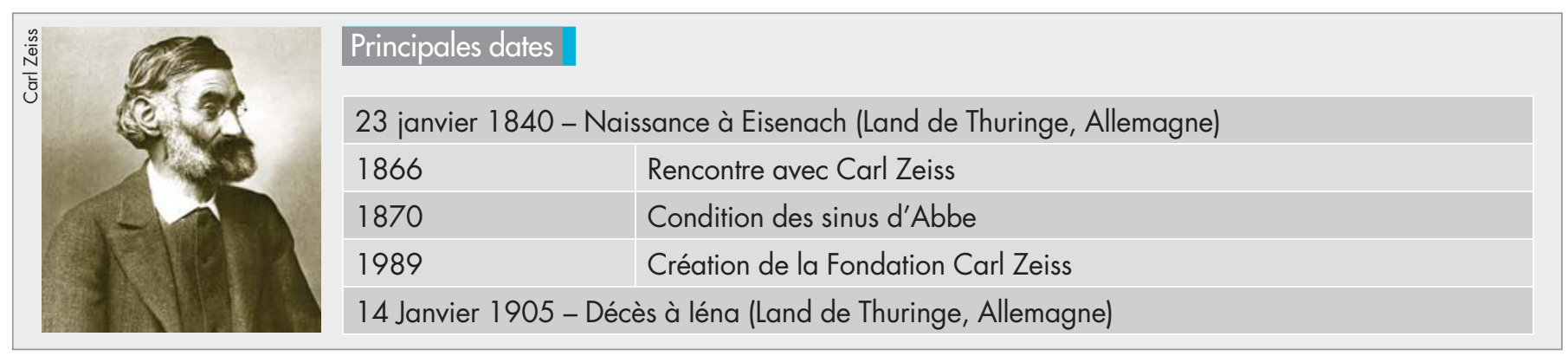

\title{
Ernst Karl Abbe
}

\author{
Riad Haidar, haidar@onera.fr
}

Physicien et industriel allemand, Ernst Karl Abbe est principalement connu pour avoir élaboré la théorie de la résolution des instruments d'optique et pour avoir établi la formule, connue désormais comme la condition des sinus d'Abbe, pour la formation d'une image nette dans un microscope. Son nom reste associé à ceux des opticiens Schott et Zeiss, et est indissociable du succès de la célèbre entreprise Carl Zeiss AG.

$E^{2}$ rnst Karl Abbe naît le 23 janvier 1840 à Eisenach (qui est aussi la ville natale de Jean-Sébastien Bach) dans le grand-duché de Saxe, alors état germanique indépendant, et actuellement Land de Thuringe au centre de l'Allemagne. Ses parents Christina et Georg Adam, ce dernier travaillant comme ouvrier dans une filature textile, sont d'une condition très modeste et la famille vit chichement.

Après l'école élémentaire, Ernst parvient toutefois à poursuivre son éducation secondaire, puis supérieure, grâce à une bourse financée par les employeurs de son père. Ce comportement de patron social l'influencera fortement lorsque, à son tour, Abbe sera capitaine d'industrie. Mais n'anticipons pas : pour l'heure, $c^{\prime}$ est un élève studieux et pugnace, doué pour les sciences. En 1857 , il décroche son Abitur avec des notes très convenables. À tout juste 17 ans, le jeune Abbe montre un caractère obstiné et fait preuve d'une volonté farouche : malgré le chagrin causé par le brusque décès de sa mère au mois de juillet et une situation financière toujours exsangue, il décide de poursuivre ses études et convainc son père de soutenir son projet.

Il intègre l'université d'léna, s'inscrit dans le cursus scientifique, et boucle ses fins de mois en donnant des cours particuliers. En 1859 , il démarre une thèse à l'université de Göttingen, et obtient en mars 1861 son doctorat de physique - option thermodynamique. II travaille ensuite quelques mois à l'observatoire de Göttingen, puis à Francfort au sein de la Physikalischer Verein, une société savante privée fondée par Johann Wolfgang von Goethe en 1824 et toujours active de nos jours. II y fait notamment la connaissance du mathématicien Michel Reiss, un généreux mécène qui lui finance ses travaux de recherche : c'est un soutien inespéré, qui permet à Abbe de décrocher son habilitation - en mathématiques, cette fois. Sa carrière académique est lancée : il devient Privatdozent en physique et mathématiques à l'université d'léna en 1863, puis professeur en 1870 ; quelques années plus tard, il dirigera l'observatoire d'léna. En 1871, il épouse Elise Snell, la fille de son professeur de physique Karl Snell. Leur fille Paula naît trois ans plus tard, en 1874 ...

Le fils pauvre d'ouvrier-fileur parvient ainsi, à force de volonté et de travail, à s'établir socialement et à creuser son sillon dans le monde. Mieux encore : il s'apprête à y laisser une empreinte indélébile, grâce à un coup de pouce du destin et à une rencontre avec un jeune industriel audacieux et visionnaire.

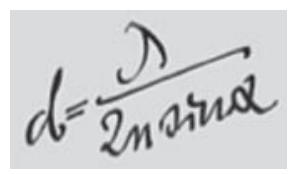

\section{L'aventure Carl Zeiss AG}

Un bref historique s'impose : en 1846, Carl Zeiss ouvre un atelier $d^{\prime}$ ingénierie de précision et d'optique à léna, où l'on fabrique en particulier des microscopes. La mise au point des combinaisons de lentilles pour les objectifs reste largement empirique et requiert $d^{\prime}$ innombrables essais et réglages manuels; c'est donc un processus hasardeux et long. Or Zeiss a la conviction que cette étape peut être formalisée, et que l'optimisation d'un système optique obéit à des principes analytiques. C'est avec cette idée en tête qu'il recrute Abbe et le nomme directeur de la recherche de son entreprise en 1866. Sous la férule pragmatique de Zeiss, Abbe élabore la théorie de la résolution des instruments d'optique, et apporte des améliorations décisives pour la conception des microscopes : il invente notamment les lentilles apochromatiques en 1868, et généralise deux ans plus tard l'utilisation du condenseur pour obtenir un champ d'observation large et uniformément lumineux. Surtout, il établit en 1870 la formule, connue désormais comme la condition 


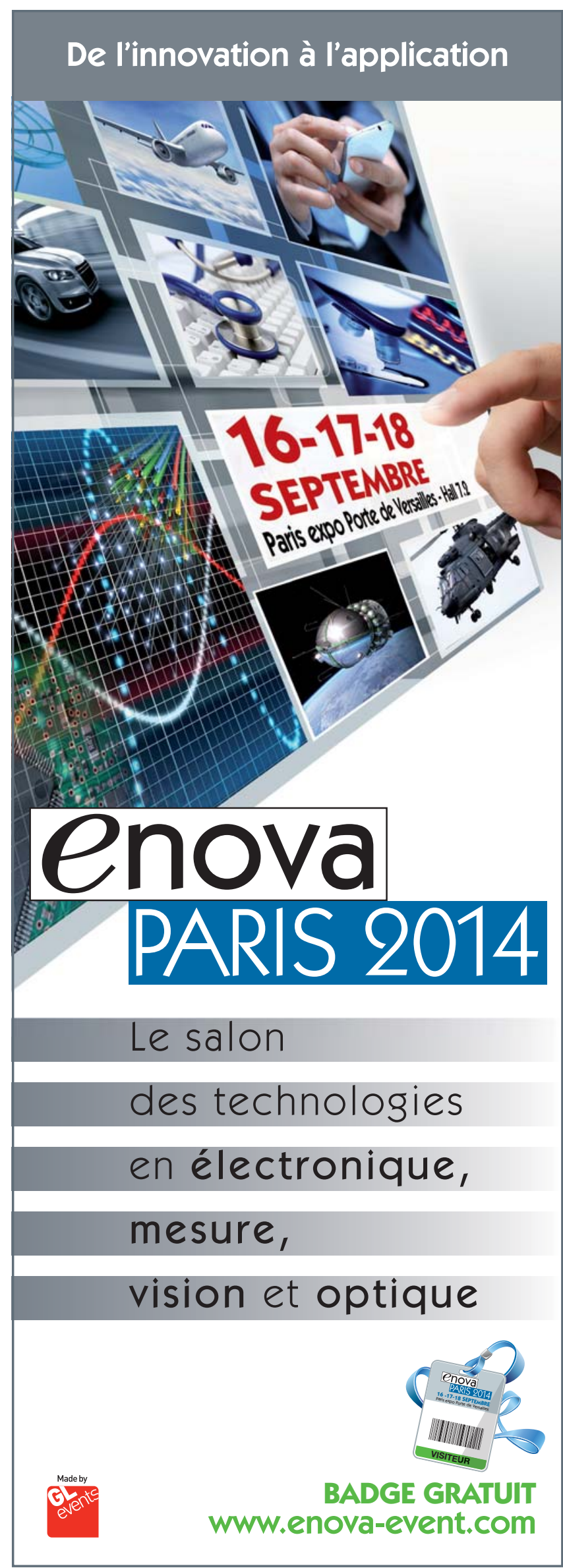

des sinus d'Abbe, pour la formation d'une image paraxiale nette (corrigée de l'aberration sphérique et du défaut de coma) dans un microscope. Zeiss, convaincu et enthousiasmé, lui cède des parts de Carl Zeiss AG en 1875.

Indiscutablement, l'entreprise jouit d'un positionnement sain. Mais il lui manque encore le matériau optimal pour fabriquer les optiques. Dès 1879 c'est chose faite, avec la rencontre d'Otto Schott, qui met au point le verre avec les caractéristiques requises. Cette collaboration tricéphale se révèle formidablement productive et confère à l'entreprise une avance technologique considérable sur ses concurrents, ce qui lui permet d'acquérir le leadership qu'on lui connaît encore aujourd'hui. Très vite, I'entreprise se diversifie et produit des jumelles, des objectifs pour caméras, ou encore des instruments pour l'astronomie ou la médecine...

Peu après la mort de Zeiss en 1888, Abbe cède son patrimoine à une fondation, qu'il baptise du nom de son associé défunt, dont les statuts imposent un lien fort entre science et technique, et qui invente avant l'heure le concept d'entreprenariat social en répartissant les profits entre la direction, les travailleurs et l'université $d^{\prime}$ léna. De toute évidence, Abbe n'a pas oublié le soutien qu'il a reçu dans sa jeunesse de la part des patrons de son père... En 1919, Schott cède à son tour son entreprise à la Fondation Carl Zeiss, qui devient de fait l'actionnaire unique de Carl Zeiss AG et de Schott AG. Ce montage, aussi original que fécond, offre aux deux entreprises une pérennité et une stabilité à toute épreuve.

\section{Les honneurs}

Ernst Abbe est libéré de sa charge d'enseignant par l'université $d^{\prime}$ léna en 1891. Parmi les nombreuses marques de reconnaissance qu'il reçoit, notons qu'il est fait en 1901 membre honoraire de l'Académie Saxonne des Sciences de Leipzig et de la Société des Sciences de Göttingen. II prend sa retraite en 1903 ; à cette occasion, en signe d'affection et de reconnaissance, les 1500 employés des entreprises gérées par la Fondation Carl Zeiss lui font une procession aux flambeaux. En 1904, il est proposé par Otto Wiener et Adolf Winkelmann pour le prix Nobel de physique - qu'il n'obtient pas... II décède le 14 janvier 1905, à 65 ans, au terme d'une longue maladie.

\section{Référence}

Kerstin Gerth, Ernst Abbe: Scientist, Entrepreneur and Social Reformer, Bussert \& Stadeler (2005).

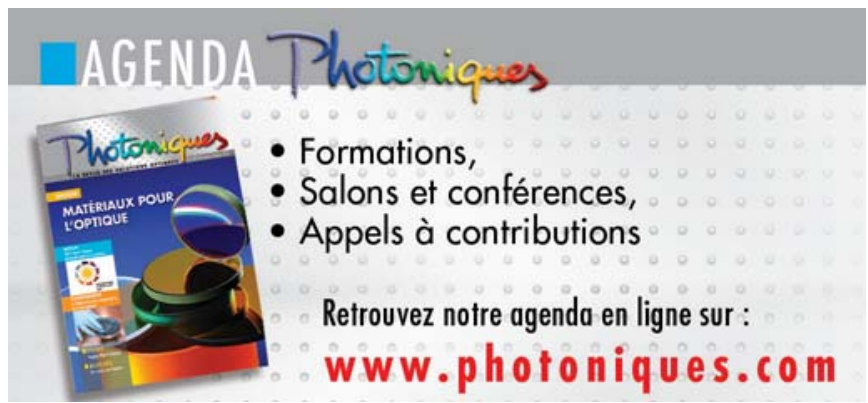

